\title{
On Existence of Eigen Values of Several Operator Bundles with Two Parameters
}

\author{
Makhmudova Malaka Gasan ${ }^{1}$, Sultanova EInara Bayram² \\ ${ }^{1}$ Department of Higher Mathematics, Baku State University, Baku, Azerbaijan \\ ${ }^{2}$ Department of Applied Mathematics, Baku State University, Baku, Azerbaijan
}

Email address:

elnare-sultanova@mail.ru (Sultanova E. B.)

\section{To cite this article:}

Makhmudova Malaka Gasan, Sultanova Elnara Bayram. On Existence of Eigen Values of Several Operator Bundles with Two Parameters. Pure and Applied Mathematics Journal. Special Issue: Spectral Theory of Multiparameter Operator Pencils and Its Applications. Vol. 4, No. 4-1, 2015, pp. 16-21. doi: 10.11648/j.pamj.s.2015040401.14

\begin{abstract}
For the several operator bundles with two parameters when the number of equation is greater than the number of parameters in the Hilbert spaces is given the criterion of existence of the common point of spectra. In the special case the common point of spectra is the common eigen value. In the proof of the theorem the authors use the results of the spectral theory of multiparameter systems.
\end{abstract}

Keywords: Operator, Space, Resultant, Criterion, Eigenvector, Bundle

\section{Introduction}

Spectral theory of multiparameter systems arose as a result of studying questions related with the solutions of partial differential equations and equations of mathematical physics. Founder of the spectral theory of multiparameter systems is F.V Atkinson. Atkinson [1] studied the available at the time fragmentary results for multiparameter symmetric differential systems, built multiparameter spectral theory of multiparameter systems of operators in finite-dimensional Euclidean spaces. Further, by taking the limit Atkinson built the spectral theory of multiparameter systems with selfadjoint compact operators in infinite-dimensional Hilbert spaces.

Later, many of his followers have studied spectral problems of self-adjoint multiparameter systems.

\section{Necessary Definitions and Notions}

Consider the two parameter system

$$
\begin{aligned}
& A_{i}(\lambda, \mu) x_{i}=\left(A_{0, i}+\lambda A_{1, i}+\ldots+\lambda^{m_{1}} A_{m_{1}}+\mu A_{m_{1}+1, i}+\ldots+\mu^{n_{1}} A_{m_{1}+n_{1}, i}\right) x_{i}=0 \\
& i=1,2, \ldots, N
\end{aligned}
$$

in the Hilbert spaces $H_{i}$;

$H=H_{1} \otimes H_{2} \otimes \ldots \otimes H_{N}$ is the tensor product of spaces $H_{1}, H_{2}, \ldots, H_{N}$

We consider the case when the number of operator equations in (1) is greater than the number of parameters. Let the number of equations in (1) is equal to $N>2$. The case when $N=2$ is considered in the work [9].

$$
\left[f_{1} \otimes \ldots \otimes f_{N}, g_{1} \otimes \ldots \otimes g_{N}\right]_{H}=\left(f_{1}, g_{1}\right)_{H_{1}} \cdot\left(f_{2}, g_{2}\right)_{H_{2}} \cdots\left(f_{N}, g_{N}\right)_{H_{N}}
$$

This definition is spread on other elements of the tensor
In (1) linear operators $A_{k, i}\left(k=0,1, \ldots, m_{i}+n_{i}\right)$ act in the Hilbert space $H_{i}$.

Let $f_{1} \otimes f_{2} \otimes \ldots \otimes f_{N} \in H \quad$ and $\quad g_{1} \otimes g_{2} \otimes \ldots \otimes g_{N} \in H \quad$ be two decomposable tensors .The inner product of these elements in the space $H_{1} \otimes H_{2} \otimes \ldots \otimes H_{N}$ is defined by means of the formulae 
continuity

Let's reduce a series of known positions concerning the spectral theory of multiparameter system.

Definition1. ([1, 3]) $(\lambda, \mu) \in C^{2}$ is an eigen value of the system (1) if there are non-zero elements $x_{i} \in H_{i}, i=1,2, \ldots, N$ such that (1) is fulfilled. A decomposable tensor $z=x_{1} \otimes \ldots \otimes x_{N}$ is named an eigenvector of the system (1), corresponding to the eigenvalue $(\lambda, \mu) \in C^{2}$.

Definition2. The operator $A_{k, i}^{+}$is induced by an operator $A_{k, i}$, acting in the space $H_{i}$, into the tensor space $H=H_{1} \otimes H_{2} \otimes \ldots \otimes H_{N}$, if on each decomposable tensor $x=x_{1} \otimes x_{2} \otimes \ldots \otimes x_{N} \quad$ of tensor product space $H=H_{1} \otimes H_{2} \otimes \ldots \otimes H_{N} \quad$ we have $A_{k, i}^{+}=E_{1} \otimes \ldots \otimes E_{i-1} \otimes A_{k, i} \otimes E_{i+1} \otimes \ldots \otimes E_{N}$ and on all the other elements of the space $H$ operator $A_{k, i}^{+}$is defined on linearity and continuity. $E_{i}$ is an identical operator in $H_{i}, i=1,2, \ldots, N$

Definition3. [5]. Let be two polynomial bundles

$$
\begin{aligned}
& A(\lambda)=A_{0}+\lambda A_{1}+\ldots+\lambda^{m} A_{m}, \\
& B(\lambda)=B_{0}+\lambda B_{1}+\ldots+\lambda^{n} B_{n},
\end{aligned}
$$

depending on the same parameter $\lambda$ and acting, generally speaking, in various Hilbert spaces $H_{1}, H_{2}$, accordingly.

The operator $\operatorname{Re} s(A(\lambda), B(\lambda))$ is given with help of matrix

$$
\begin{aligned}
& \operatorname{Re} s(A(\lambda), B(\lambda))=
\end{aligned}
$$

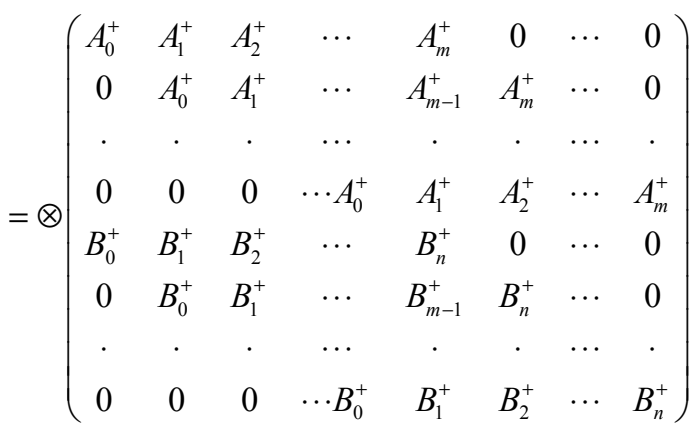

In $[4,11]$ this operator is called an abstract analog of an Resultant for polynomial bundles (2).

In definition of a Resultant (3) of bundles (2) lines with operators $A_{i}$ repeated $n$ times, and lines with operators $B_{i}$ repeated exactly $m$ times Numbers $m, n$ are the greatest degrees of parameter $\lambda$ in bundles $A(\lambda)$ and $B(\lambda)$ accordingly. Thus, the Resultant is an operator, acting in space $\left(H_{1} \otimes H_{2}\right)^{m+n}$ that is a direct sum $m+n$ of copies of tensor product spaces $H_{1} \otimes H_{2}$. Value of $\operatorname{Re} s(A(\lambda), B(\lambda))$ is equal to its formal expansion when each term of this expansion is tensor product of operators. Let operator $A_{m}$, or $B_{n}$ is invertible. If the greatest degrees of parameter $\lambda$ in bundles of $A(\lambda)$ and $B(\lambda)$ coincide (see [11]) or if the greatest degrees of parameter $\lambda$ in bundles of $A(\lambda)$ and $B(\lambda)$, generally speaking, can not coincide (see [4]) bundles (2) have a common eigen values in the only case when the Resultant of these bundles has non-zero kernel. In fact, that in case of when bundles act in finite-dimensional spaces, common point of spectra of these bundles is a common eigen value.

When in (1) the number $N=2$ the following result is proved in the work [9]:

Theorem1. Let operators $A_{i}\left(i=0,1, \ldots, m_{1}+n_{1}\right)$ also $B_{i}\left(i=0,1, \ldots, m_{2}+n_{2}\right)$ act in finite-dimensional spaces $H_{1}$ and $H_{2}$, accordingly, and one of three following conditions is fulfilled:

a) $\max \left(m_{1} n_{2}, m_{2} n_{1}\right)=m_{1} n_{2}, \operatorname{Ker} A_{m_{1}}=\{\theta\}, \operatorname{Ker} B_{m_{2}+n_{2}}=\{\theta\}$; $A_{m_{1}}, B_{m_{2}+n_{2}}$ are selfadjoint operators everyone in their space

b) $\max \left(m_{1} n_{2}, m_{2} n_{1}\right)=m_{2} n_{1}, \operatorname{Ker} B_{m_{2}}=\{\theta\}, \operatorname{Ker} A_{m_{1}+n_{1}}=\{\theta\}$ operators $B_{m_{2}} A_{m_{1}+n_{1}}$ are selfadjoint everyone in their spaces

$$
m_{1} n_{2}=m_{2} n_{1}
$$

$\operatorname{Ker}\left(A_{m_{1}}^{n_{2}} \otimes B_{m_{2}+n_{2}}^{n_{1}}+(-1)^{n_{1} n_{2}} A_{m_{1}+n_{1}}^{n_{2}} \otimes B_{m_{2}}^{n_{1}}\right)=\{\theta\}$

$A_{m_{1}}, B_{m_{2}}, A_{m_{1}+n_{1}}, B_{m_{2}+n_{2}}$ are the self-adjoint operators, acting everyone in its finite-dimensional space.

Then the $\max \left(m_{1} n_{2}, m_{2} n_{1}\right)$-multiple basis of system of eigen and associated vectors of (2) takes place

This paper is devoted to the investigation of the system (1) when the $N>2$. The goal of our investigations is the finding the criterion of existing of common point of spectra of the system (1).

For proving we use the notion of criterion of existence of common point of spectra of several bundles with the same one parameter.

\section{Necessary and Sufficient Conditions of Existence of Eigen Values of Several Operator Polynomials}

Consider [7,10]

$$
\left\{\begin{array}{l}
B_{i}(\lambda)=B_{0, i}+\lambda B_{1, i}+\ldots+\lambda^{k_{i}} B_{k_{i}, i} \\
i=1,2, \ldots, n
\end{array}\right.
$$

when $B_{i}(\lambda)$ is an operator bundle with a discrete spectrum, acting in Hilbert space $H_{i}(i=1,2, \ldots, n)$. Without loss of generality, we assume that. $k_{1} \geq k_{2} \geq \ldots \geq k_{n} \cdot H^{k_{1}+k_{2}}$ is the direct sum of $k_{1}+k_{2}$ copies of the tensor spaces $H=H_{1} \otimes \ldots \otimes H_{n}$.

Introduce the operators $R_{i}(i=1,2, \ldots, n-1)$ with help of the operator matrices (5) 


$$
\begin{aligned}
& R_{i-1}= \\
& =\left(\begin{array}{cccccccc}
B_{0,1}^{+} & B_{1,1}^{+} & \cdots & B_{k_{1}, 1}^{+} & 0 & 0 & \cdots & 0 \\
0 & B_{0,1}^{+} & \cdots & B_{k_{1}-1,1}^{+}, & B_{k_{1}, 1}^{+} & 0 & \cdots & 0 \\
\cdot & \cdot & \cdots & \cdot & \cdot & \cdot & \cdots & \cdot \\
\cdot & \cdot & \cdots & B_{0,1}^{+} & B_{1,1}^{+} & \cdot & \cdots & B_{k_{1}, 1}^{+} \\
B_{0, i}^{+} & B_{1, i}^{+} & \cdots & B_{k_{i}-1, i}^{+} & B_{k_{i}-1, i}^{+} & 0 & \cdots & 0 \\
0 & B_{0, i}^{+} & \cdots & B_{k_{i}-2, i}^{+} & B_{k_{i}-1, i}^{+} & B_{k_{i}, i}^{+} & \cdots & 0 \\
\cdot & \cdot & \cdots & \cdot & \cdot & \cdot & \cdots & \cdot \\
0 & 0 & 0 & 0 & B_{0, i}^{+} & B_{1, i}^{+} & \cdots & B_{k_{i}, i}^{+}
\end{array}\right)
\end{aligned}
$$

$$
\bigcap_{i=2}^{n} \operatorname{KerR}_{i} \neq\{\theta\},\left(\operatorname{Ker} B_{k_{1}}=\{\theta\}\right)
$$

This result is obtained in [10].

Rows with the operators $B_{i, 1}^{+}\left(i=0,1, \ldots, k_{1}\right)$ are repeated $k_{2}$ times and rows with the operators $B_{s, i}^{+}\left(s=0,1, \ldots, k_{2}\right), B_{k_{i}+1, i}=\ldots=B_{k_{2}, i}=0 \quad$ are $\quad$ repeated $\max \left(m_{1} n_{2}, m_{2} n_{1}\right)=m_{2} n_{1} \quad$ times. The operators $B_{i, s}^{+}\left(i=1,2, \ldots, k_{s} ; s=2, \ldots, k_{i}\right)$ are induced by an operator $B_{i, s}$, acting in the space $B_{i, s}$, into the space $H_{1} \otimes \cdots \otimes H_{N}$ by the formulae

$$
B_{i, s}^{+}=E_{1} \otimes \cdots \otimes E_{s-1} \otimes B_{i, s} \otimes E_{s+1} \otimes \cdots \otimes E_{n}
$$

Denote $\sigma_{p}\left(B_{i}(\lambda)\right)$ the set of eigen values of operator $B_{i}(\lambda)$.

Theorem 2.[10]. Let all operators $B_{j, k}$ are bounded in the corresponding spaces $H_{k}$, the operator $B_{k_{1}, 1}$ has an inverse. Spectrum of each operator pencil $B_{i}(\lambda)$ contains only eigen values.

$$
\text { Then } \bigcap_{i=1}^{n} \sigma_{p}\left(B_{i}(\lambda)\right) \neq\{\vartheta\} \text { if and only if }
$$

\section{Criterion of Existence of Common Eigen Values of Two Polynomial Bundles with Two Parameters}

We fix in the all operators (1) the parameter $\lambda$. Let be

$\lambda=\lambda_{0}$. Then we have $N$ operators, depending on the same parameter $\mu$. Without loss of generality we suppose $n_{1} \geq n_{2} \geq \ldots \geq n_{N}$.

Then the system (1) turns out to the system

$$
\begin{aligned}
& A_{i}\left(\mu\left(\lambda_{0}\right)\right) x_{i}=\left(A_{0, i}+\lambda_{0} A_{1, i}+\ldots+\lambda_{0}{ }^{m_{1}} A_{m_{1}}+\right. \\
& \left.+\mu A_{m_{1}+1, i}+\ldots+\mu^{n_{1}} A_{m_{1}+n_{1}, i}\right) x_{i}=0 \\
& i=1,2, \ldots, N
\end{aligned}
$$

So the parameter $\lambda$ is fixed arbitrarily we missed the index 0 in the $\lambda_{0}$. Denote though $A_{i}^{+}(\mu(\lambda))$ the operator in the space $H$, induced into $H$ by the operator $A_{i}(\mu(\lambda))$ acting in the space $H_{i}$.

Let all operators $A_{m_{i}+n_{i}, i}^{-1}, i=1,2, \ldots, N$ exist and bounded. Introduce the notations

$$
\begin{aligned}
& \tilde{A}_{i}(\lambda)=A_{0, i}+\lambda A_{1, i}+\ldots+\lambda^{m_{i}} A_{m_{i}, i} \\
& i=1,2, \ldots, N
\end{aligned}
$$

Construct the resultants of operators $A_{1}(\mu(\lambda))$ and $A_{i}(\mu(\lambda)), i=2, \ldots, N$.

$$
\begin{aligned}
& R_{i-1}=\left(\begin{array}{cccccccc}
\tilde{A}_{1}^{+}(\lambda) & A_{m_{1}+1,1}^{+} & \cdots & A_{m_{1}+n_{1}, 1}^{+} & 0 & 0 & \cdots & 0 \\
0 & \tilde{A}_{1}^{+}(\lambda) & \cdots & A_{m_{1}+n_{1}-1,1}^{+} & A_{m_{1}+n_{1}, 1}^{+} & 0 & \cdots & 0 \\
\cdot & \cdot & \cdots & \cdot & \cdot & \cdot & \cdots & \cdot \\
\cdot & \cdot & \cdots & \tilde{A}_{1}^{+}(\lambda) & A_{m_{1}+1,1}^{+} & \cdot & \cdots & A_{m_{1}+n_{1}, 1}^{+} \\
\tilde{A}_{i}^{+}(\lambda) & A_{m_{i}+1, i}^{+} & \cdots & A_{m_{i}+n_{i}-1, i}^{+} & A_{m_{i}+n_{i}, i}^{+} & 0 & \cdots & 0 \\
0 & \tilde{A}_{i}^{+}(\lambda) & \cdots & A_{m_{i}+n_{i}-2, i}^{+} & A_{m_{i}+n_{i}-1, i}^{+} & A_{m_{i}+n_{i}, i}^{+} & \cdots & 0 \\
\cdot & \cdot & \cdots & \cdot & \cdot & \cdot & \cdots & \cdot \\
0 & 0 & 0 & 0 & \tilde{A}_{i}^{+}(\lambda) & A_{m_{i}+1, i}^{+} & \cdots & A_{m_{i}+n_{2}, i}^{+}
\end{array}\right) \\
& i=2, \ldots, N
\end{aligned}
$$

The rows in (8) with the operators $\tilde{A}_{1}^{+}(\lambda), A_{m_{1}+1,1}^{+}, \ldots, A_{m_{1}+n_{1}, 1}^{+}$ are repeated $n_{2}$ time, rows with the operators $\tilde{A}_{i}^{+}(\lambda), A_{m_{i}+1, i}^{+}, \ldots, A_{m_{i}+n_{1}, i}^{+}$are repeated $n_{1}$ time for all meanings of $i$. The operators $R_{i}$ act in the direct sum of $n_{1}+n_{2}$ copies of tensor product space $H=H_{1} \otimes \ldots \otimes H_{N}$. If
$\operatorname{Ker}_{m_{i}+n_{i}, i}=\{\vartheta\} i=1,2, \ldots, N, \quad, \operatorname{Ker} R_{i}=\{\vartheta\}$, all operators $A_{m_{1}+n_{1}, 1}^{-1} \quad, A_{m_{i}+n_{i}, i}^{-1} \quad \tilde{A}_{i}(\lambda), A_{m_{i}+1, i}, \ldots, A_{m_{i}+n_{i}, i}$ are bounded for all meanings of $i$. Suppose that the eigen and associated vectors of the bundle $A_{1}(\mu(\lambda))$ for each fixed $\lambda$ form the basis of the space $H_{1}$. Consider the operators $R_{i}$. It is known they act in the direct sum of $n_{1}+n_{2}$ copies of tensor product 
space $H=H_{1} \otimes \ldots \otimes H_{N}$. From the results of the spectral theory of multiparameter system the first component of the element of the kernel of $R_{i}$ is the linear combination of elements of an aspect

$$
U_{i, 1} \otimes E_{2} \otimes \ldots \otimes E_{i-1} \otimes U_{0, i} \otimes \ldots \otimes E_{N}+U_{i-1,1} \otimes E_{2} \otimes \ldots \otimes U_{1, i} \otimes \ldots \otimes E_{N}+\ldots+U_{0,1} \otimes \ldots \otimes U_{i, i} \otimes \ldots \otimes E_{N},
$$

when $U_{0.1}, U_{1,1}, \ldots, U_{s, 1}\left(\left(\right.\right.$ accordingly, $\left.U_{0, i}, U_{1, i}, \ldots, U_{s, i}\right)$ there is a restricted chain of eigen and associated vectors of an operator $A_{1}(\mu(\lambda))$ (accordingly, $A_{j}(\mu(\lambda))$ ), corresponding to some common eigenvalue $\mu(\lambda)$ of both operators. It is clear if $\operatorname{Ker} R_{i} \neq\{\vartheta\}$ and all other conditions are fulfilled the element of the type $\sum_{i+j=s} U_{0, i} \otimes E_{2} \otimes \ldots \otimes E_{i-1} \otimes U_{0, j} \otimes \ldots \otimes E_{N}, s=0,1, \ldots \quad$ form the first component of the element entering the kernel of the resultant of operator bundles $A_{1}(\mu(\lambda))$ and $A_{j}(\mu(\lambda))$

If the condition $\operatorname{Ker} \cap R_{i}(\lambda) \neq\{\vartheta\}$ is fulfilled and the element $Z \in \operatorname{Ker} \cap R_{i}(\lambda), 0 \neq Z \in H^{n_{1}+n_{2}}$ then this element $0 \neq Z \in H^{n_{1}+n_{2}}$ enters the kernel of resultant of operator bundles $A_{1}(\mu(\lambda))$ and $A_{j}(\mu(\lambda))$ for the first component of the element $0 \neq Z \in H^{n_{1}+n_{2}}$ expression (9) are fulfilled. From [12] it follows that element of the space $H$ entering the $\operatorname{Ker} \cap R_{i}(\lambda) \neq\{\vartheta\}$ is the linear combination of elements of the aspect

$$
\sum_{i_{1}+\ldots+i_{N}=s} U_{i_{1}, 1} \otimes U_{i_{2}, 2} \otimes \ldots \otimes U_{i_{N}, N}
$$

when $U_{0.1}, U_{1,1}, \ldots, U_{s, 1}$ (accordingly, $U_{0, i}, U_{1, i}, \ldots, U_{s, i}$ ) there is a restricted chain of e.a. vectors of an operator $A_{1}(\mu(\lambda))$ and operator $A_{i}(\mu(\lambda)) \quad i=2, \ldots, N$, correspondingly ,

Naturally, when $s=0$ in (10) decomposable tensor $U_{0,1} \otimes \ldots \otimes U_{0, i} \otimes \ldots \otimes U_{0, N}$ is the first component of the element of $\operatorname{Ker} \cap R_{i}(\lambda) \neq\{\vartheta\}$ which has the form

$$
\left(\quad U_{0,1} \otimes \ldots \otimes U_{0, i} \otimes \ldots \otimes U_{0, N}, \quad \mu U_{0,1} \otimes \ldots \otimes U_{0, N}, \ldots\right.
$$
..,$\left.\mu^{N-1} U_{0,1} \otimes \ldots \otimes U_{0, N}\right)$. Element $U_{0,1} \otimes \ldots \otimes U_{0, i} \otimes \ldots \otimes U_{0, N}$ is the eigen vector of the system (1), corresponding to the eigen value $(\lambda, \mu(\lambda))$.

Denote though $\tilde{R}_{i}$ the decompositions of the resultants $R_{i}$. In fact, $\tilde{R}_{i}(i=1,2, \ldots, N-1)$ are the operator bundles, acting in the space $H=H_{1} \otimes \ldots \otimes H_{N}$.

We have the $N-1$ operator bundles with one parameter $\lambda$. The greatest degree of parameter $\lambda$ in the operator bundle $\tilde{R}_{i}(i=1,2, \ldots, N-1)$ is equal to $\max \left(m_{1} n_{2}, m_{i+1} n_{1}\right)$. Moreover, we have the following result:

Let be all operators $A_{i, k}$ bounded in the corresponding space $H_{k}, \operatorname{Ker} A_{m_{1}+n_{1}, 1}=\{\vartheta\}, \operatorname{Ker} A_{m_{i}+n_{i}, i}=\{\vartheta\}$ then the $N$ operator bundles (7), depending on the parameter $\lambda$ have the common point of spectra if and only if

$$
\bigcap_{i=1}^{N-1} \operatorname{KerR}_{i}(\lambda) \neq\{\theta\} .
$$

Remark1. If spectrum of each operator bundle in (7) at each fixed meaning of the parameter $\lambda$ contains only eigen values then the common point of spectra of these operator bundles is their common eigenvalue.

Remark 2. If the spaces $H_{k}$ are finite dimensional spaces, then this common point of spectra of (7) is the common eigenvalue of (7).

So we have the $N-1$ operator bundles $R_{i}(i=1,2, \ldots, N-1)$, depending on one parameter $\lambda$ and acting in the space $H$.

Without loss of generality we suppose

$$
\begin{aligned}
& \max \left(m_{1} n_{2}, m_{2} n_{1}\right) \geq \max \left(m_{1} n_{2}, m_{3} n_{1}\right) \geq \ldots \geq \\
& \geq \max \left(m_{1} n_{2}, m_{N} n_{1}\right)
\end{aligned}
$$

Denote the operator coefficients of the parameter $\lambda^{s}$ in the $\tilde{R}_{i}$ though $\vec{A}_{s, i} . \max \left(m_{1} n_{2}, m_{i+1} n_{1}\right)=M_{i}, i=1,2, \ldots, N-1$

All operators $\vec{A}_{s, i}$ act in the space $H$.

Thus we have $N-1$ operator bundles, acting in the space $H$ and depending on one same parameter:

$$
\begin{gathered}
\tilde{R}_{i}=\dddot{A}_{0, i}+\lambda \dddot{A}_{1, i}+\cdots+\lambda^{M_{i}} \dddot{A}_{M_{i}, i} \\
i=1, \ldots, N-1
\end{gathered}
$$

Further, the proof is spent by analogy of the proof the Theorem2. Construct the resultants of the obtained operator bundles $\tilde{R}_{1}$ and $\tilde{R}_{2}, \tilde{R}_{1}$ and $\tilde{R}_{3}, \ldots, \tilde{R}_{1}$ and $\tilde{R}_{N-1}$.

The resultants have the form

$$
\begin{aligned}
& \tilde{\tilde{R}}_{i-1}=
\end{aligned}
$$

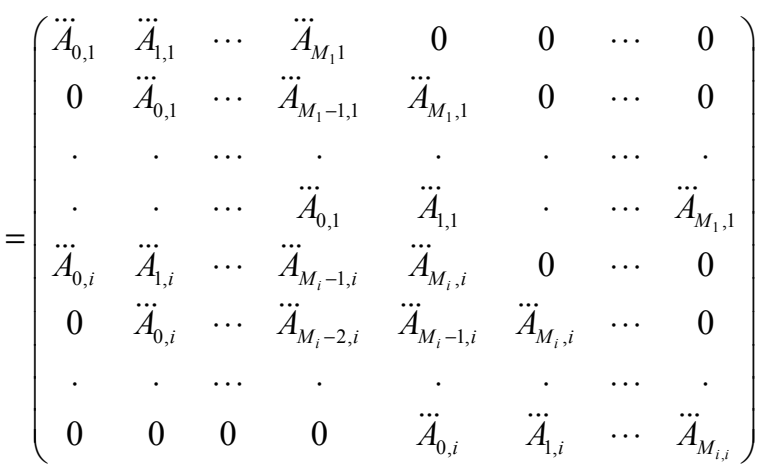

$$
\begin{aligned}
& i=2, \ldots, N-1
\end{aligned}
$$

In the resultant $\tilde{\tilde{R}}_{i-1}$ the rows with the operators $\dddot{A}_{0,1}, \dddot{A}_{1,1}, \ldots, \dddot{A}_{M_{1}, 1}$ are repeated $\max \left(m_{1} n_{2}, m_{3} n_{1}\right)$ times, and the 
rows with the operators $\dddot{A}_{0, i}, \dddot{A}_{1, i}, \ldots, \dddot{A}_{M_{i}, i}$ are repeated $\max \left(m_{1} n_{2}, m_{2} n_{1}\right)$ times.

Theorem 3. The system of operator bundles (1) have a common points of spectra if and only if $\operatorname{Ker} \bigcap_{i=1}^{N-2} \tilde{\tilde{R}}_{i} \neq\{\vartheta\}$ $\operatorname{Ker} \bigcap_{i=1}^{N-1} R_{i} \neq\{\vartheta\}, \operatorname{Ker} \dddot{A}_{M_{i}, i 1}=\{\vartheta\}, \operatorname{Ker} A_{m_{i}+n_{i}, i}=\{\vartheta\}$, all operators, forming the system (1) and $A_{m_{i}+n_{i}, i}^{-1}, \dddot{A}_{M_{i}, i}^{-1}$ are bounded in corresponding spaces.

Proof of the Theorem 3.

Necessity. Let the system (1) has a common point $(\lambda, \mu)$ of spectra. Then for fixed first component of $(\lambda, \mu)$ the spectrum of each operator bundle contains only eigen values . From the Theorem1 it follows that $\bigcap_{i=1}^{N-1} \operatorname{Ker} R_{i} \neq\{\theta\}$. Last means that the system (12) has the eigen value $\lambda$ and there is the non-zero decomposable tensor of the space $H$ that following equations (13)

$$
\tilde{R}_{i} z=\left(\dddot{A}_{0, i}+\lambda \dddot{A}_{1, i}+\cdots+\lambda^{M_{i}} \dddot{A}_{M_{i}, i}\right) z=0
$$

$i=1, \ldots, N-1, \quad z=x_{1} \otimes x_{2} \otimes \ldots \otimes x_{N}$ are satisfied.

So (13) is fulfilled then the result of theorem2 demands the fulfilling of the condition $\operatorname{Ker} \bigcap_{i=1}^{N-2} \tilde{\tilde{R}}_{i} \neq\{\vartheta\}$.

Sufficiency. Let $\quad$ be $\bigcap_{i=1}^{N-1} \operatorname{KerR}_{i} \neq\{\theta\}, \quad \operatorname{Ker} \bigcap_{i=1}^{N-2} \tilde{\tilde{R}} \neq\{\vartheta\}$ $\operatorname{Ker} \dddot{A}_{M_{i}, i 1}=\{\vartheta\}, \operatorname{Ker}_{m_{i}+n_{i}, i}=\{\vartheta\}$, operators $A_{m_{i}+n_{i}, i}^{-1}$ and $\dddot{A}_{M_{i}, i}^{-1}$ are bounded.

Let be $\bigcap_{i=1}^{N-1} \operatorname{Ker}_{i} \neq\{\theta\}$. Last means there is non-zero element of the space $H^{n_{1}+n_{2}}$ (direct sum of the $n_{1}+n_{2}$ copies of the space $H$ ), entering $R_{i}$. Each operator $R_{i}$ is the resultant of the bundles $A_{1}(\mu(\lambda))$ and $A_{i+1}(\mu(\lambda))$ From $\bigcap_{i=1}^{N-1} \operatorname{Ker} R_{i} \neq\{\theta\}$ it follows the (12) are satisfied. Therefore , each equation in (13) means that the first operator bundle with the any other operator bundle has the common eigen value $\mu(\lambda)$ and common for all operator bundles eigenvector. $\mu(\lambda)$ is the common eigen value of the system(1) for this fixed $\lambda$.

All operators $\tilde{\tilde{R}}_{i}$ act in the same Hilbert space- direct sum of $\max \left(m_{1} n_{2}, m_{2} n_{1}\right)+\max \left(m_{1} n_{2}, m_{3} n_{1}\right)$ copies of the space $H$.

The condition $\operatorname{Ker} \bigcap_{i=1}^{N-1} \tilde{\tilde{R}}_{i} \neq\{\vartheta\}$ means that the results of the theorem2 take place for the system (1). Consequently, the system (1) has a common eigen value.

Theorem 3 is proved.

\section{Nonlinear Algebraic System of Equations with Two Unknown Variables}

Consider the algebraic system with two variables, when the number of equations is greater than 2 .

$$
\begin{aligned}
& a_{i}(x, y)=a_{0, i}+a_{1, i} x+\ldots+a_{m_{1}, i} x^{m_{i}}+a_{m_{1}+1, i} y+\ldots+a_{m_{1}+n_{1}, i} y^{n_{i}}=0 \\
& i=1,2, \ldots, N
\end{aligned}
$$

Instead of the spaces $H_{i}$ we adopt the space $R$ and the variables $x, y$ play the role of the parameters $\lambda, \mu$, correspondingly. Numbers $a_{i, k}$ are the bounded operators in

$$
\begin{aligned}
& R_{i-1}= \\
& \left(\begin{array}{cccc}
a_{1}(x) & a_{m_{1}+1,1} & \cdots & a_{m_{1}+n_{1}, 1} \\
0 & a_{1}(x) & \cdots & a_{m_{1}+n_{1}-1,1} \\
\cdot & \cdot & \cdots & \cdot \\
\cdot & \cdot & \cdots & a_{1}(x) \\
a_{i}^{+}(x) & a_{m_{i}+1, i}^{+} & \cdots & a_{m_{i}+n_{i}-1, i} \\
0 & a_{i}(x) & \cdots & a_{m_{i}+n_{i}-2, i} \\
\cdot & \cdot & \cdots & \cdot \\
0 & 0 & 0 & 0
\end{array}\right. \\
& i=2, \ldots, N
\end{aligned}
$$

when $a_{i}(x)=a_{0, i}+a_{1, i} x+\ldots+a_{m_{1}, i} x^{m_{i}}, i=1,2, \ldots, N$ the space $R$.

The operators $R_{i}$ and $\tilde{\tilde{R}}$ turn out to the form

$$
\left.\begin{array}{cccc}
0 & 0 & \cdots & 0 \\
a_{m_{1}+n_{1}, 1}^{+} & 0 & \cdots & 0 \\
\cdot & \cdot & \cdots & \cdot \\
a_{m_{1}+1,1} & \cdot & \cdots & a_{m_{1}+n_{1}, 1} \\
a_{m_{i}+n_{i}, i} & 0 & \cdots & 0 \\
a_{m_{i}+n_{i}-1, i} & a_{m_{i}+n_{i}, i} & \cdots & 0 \\
\cdot & \cdot & \cdots & \cdot \\
a_{i}(x) & a_{m_{i}+1, i} & \cdots & a_{m_{i}+n_{2}, i}
\end{array}\right)
$$

Denote the coefficients at $x^{s}$ in decomposition $R_{i}$ though 
$\tilde{a}_{s, i}$. So we obtain $N-1$ polynomials

$$
\begin{aligned}
& R_{i}=\tilde{a}_{0, i}+\tilde{a}_{1, i} x+\cdots+\tilde{a}_{M_{i}, i} x^{M_{i}} \\
& i=1,2, \ldots, N-1
\end{aligned}
$$

Accord to our notations we construct the operators

$$
\begin{aligned}
& \tilde{\tilde{R}}_{i-1}= \\
& =\left(\begin{array}{cccccccc}
\tilde{a}_{0,1} & \tilde{a}_{1,1} & \cdots & \tilde{a}_{M_{1} 1} & 0 & 0 & \cdots & 0 \\
0 & \tilde{a}_{0,1} & \cdots & \tilde{a}_{M_{1}-1,1} & \tilde{a}_{M_{1}, 1} & 0 & \cdots & 0 \\
\cdot & \cdot & \cdots & \cdot & \cdot & \cdot & \cdots & \cdot \\
\cdot & \cdot & \cdots & \tilde{a}_{0,1} & \tilde{a}_{1,1} & \cdot & \cdots & \tilde{a}_{M_{1}, 1} \\
\tilde{a}_{0, i} & \tilde{a}_{1, i} & \cdots & \tilde{a}_{M_{i}-1, i} & \tilde{a}_{M_{i}, i} & 0 & \cdots & 0 \\
0 & \tilde{a}_{0, i} & \cdots & \tilde{a}_{M_{i}-2, i} & \tilde{a}_{M_{i}-1, i} & \tilde{a}_{M_{i}, i} & \cdots & 0 \\
\cdot & \cdot & \cdots & \cdot & \cdot & \cdot & \cdots & \cdot \\
0 & 0 & 0 & 0 & \tilde{a}_{0, i} & \tilde{a}_{1, i} & \cdots & \tilde{a}_{M_{i, i}}
\end{array}\right) \\
& i=2, \ldots, N \\
& \hline=1
\end{aligned}
$$

Operator $\tilde{\tilde{R}}_{i}$ is the resultant of polynomials, obtaining as result of decompositions of the $R_{1}$ and $R_{i}$.

Criterion of existence of solution of the system (14) is defined by the

Theorem 4 . The system of polynomials (12) has a common solutions if and only if $\tilde{a}_{M_{i}, i} \neq 0, a_{m_{i}+n_{i}, i} \neq 0$

$$
\operatorname{Ker} \bigcap_{i=1}^{N-2} \tilde{\tilde{R}}_{i} \neq\{\vartheta\}, \operatorname{Ker} \bigcap_{i=1}^{N-1} R_{i} \neq\{\vartheta\} .
$$

\section{Conclusion}

In this paper the necessary and sufficient conditions of existence of the common eigenvalue of the two-parameter system of operators in the Hilbert space in the case when the number of operators is greater than the number of parameters are proved. As corollary of this result for the non-linear algebraic system of equations is solved the problem of existence of solutions.

\section{References}

[1] Atkinson F.V. Multiparameter spectral theory. Bull.Amer.Math.Soc.1968, 74, 1-27.

[2] Browne P.J. Multiparameter spectral theory. Indiana Univ. Math. J,24, 3, 1974.

[3] Sleeman B.D. Multiparameter spectral theory in Hilbert space. Pitnam Press, London, 1978, p.118.

[4] Balinskii A.I Generation of notions of Bezutiant and Resultant DAN of Ukr. SSR, ser.ph.-math and tech. of sciences, 1980,2. (in Russian).

[5] Dzhabarzadeh R.M. Spectral theory of two parameter system in finite-dimensional space. Transactions of NAS Azerbaijan, v. 3-4 1998, p.12-18.

[6] Dzhabarzadeh R.M. Spectral theory of multiparameter system of operators in Hilbert space, Transactions of NAS of Azerbaijan, 1-2, 1999, 33-40.

[7] Dzhabarzadeh R.M. Multiparameter spectral theory. Lambert Academic Publishing, 2012, pp. 184 (in Russian).

[8] Dzhabarzadeh R.M. Nonlinear algebraic systems. Lambert Academic Publishing, 2013, pp. 101(in Russian).

[9] Dzhabarzadeh R.M. About Solutions of Nonlinear Algebraic System with Two Variables. Pure and Applied Mathematics Journal,vol. 2, No. 1, pp. 32-37, 2013.

[10] Dzhabarzadeh R.M. On existence of common eigenvalues of some operator bundles polynomial depending on parameter. Baku, International Conference on Topoloji. 3-9 0ctober 1987.Tez.p-.2, Baku,p,93.

[11] Khayniq Q. Abstract analog of Resultant for two polynomial bundles Functional analyses and its applications, 1977, 2,no. 3, p.94-95.

[12] Dzhabarzadeh R.M. Structure of eigen and associated vectors of not adjoint multiparameter system in the Hilbert space. Proc.of IMM of NAS of Azerb.- 2011, vol.XXXV (XLIII).p.11- 21. 